benefit. The writer did not see her again, but an interview with her daughter revealed the fact that she had blebs on her eyes about three years before she died (Feb. 1, 1910), and films formed over her eyes until she became totally blind. About one year before she died, blebs appeared over the entire body, and one on the foot became septic, which was the immediate cause of her death. About two years elapsed before the eyes were involved, and over four before the skin was affected.

The diagnosis was made by eliminating the following diseases:

(1) Syphilis. No history. Blebs not characteristic of syphilis. No loss of tissue or scars characteristic of syphilis.

(2) Lupus. This disease is not one with bleb manifestations, nor do the lesions appear suddenly and then heal spontaneously in a comparatively short time. Scars peculiar to this disease were absent in this case. No nodules of granulation tissue.

(3) Diphtheria. Absence of Klebs-Lœffler bacillus and duration of the disease.

(4) Leukoplakia. Consists of thickening of the epithelium which is usually very adherent, and the lesions are not ushered in with bleb formation. It has no reddened tissue under the epithelium unless there has been some acute irritation of the part. It is not transitory.

(5) Mercurial stomatitis. No history of having taken mercury in any form. No salivation or breath that is so often found in these cases. No tenderness of teeth when mouth is suddenly closed.

(6) Herpes. Usually found in small clusters, confining itself to one side of the soft palate, uvula or pillars of the fauces. Small and round and scarcely ever assumes the size and irregular shape that is so common in pemphigus. Often associated with herpes is acute pain, while pemphigus simply is uncomfortable. Herpes lasts usually only a short time and then disappears, only to appear later.

(7) Epidermolysis bullosa hereditaria. This is the the most difficult of all to eliminate, and, indeed, if we had not the history and skin lesions to help us, I doubt if it would be possible to distinguish one from the other except from location. In the one case which the author has seen of epidermolysis, the skin lesions appeared only at points of contact or trauma, and in like manner the lesions in the mouth follow the same rule. The lesions in pemphigus seem to appear on any part of the mucous membrane without reference to trauma.

\section{ACUTE TOXEMIA CAUSED BY INFECTION OF THE THROAT BY THE COLON BACILLUS.*}

\footnotetext{
BY CALVIN B. FAUNCE, JR., M.D., BOston.

(From the Pathological Laboratory of the Massachusetts Charitable Eye and Ear Infirmary.)
}

THIs case is of unusual interest because of the rarity of colon infection in general, and especially because of the very unusual site in which the lesion here occurred.

Mrs. P., who had been in her usual good health, developed suddenly headache, chilly sensations, lumbar and joint pains. She did not complain of the throat. Twelve hours after the attack began, she suffered marked prostration, severe headache and high fever (temperature, 103). The throat was red and edematous throughout, both tonsils were much enlarged and a grayish-white exudate presented at the crypts.

* Read at a meeting of the New England Otological and Laryngological Society, Feb. 3, 1911 .
Her condition became steadily worse, so that in twentyfour hours after the onset of the disease the prostration was profound. The exudate on the tonsils had coalesced so that they both were completely covered. This exudate could be easily stripped off without bleeding. There was, in addition, a general herpetic ulcerative condition of the mouth. Cultures taken at this time were pronounced negative in regard to the presence of Klebs-Lœffler's bacillus, but the clinical condition was considered so suggestive of diphtheria that 6,000 units of antitoxin were administered.

Twelve hours after receiving the antitoxin the general condition had improved, the condition of the throat remained the same. Cultures taken at this time again failed to show the presence of the KlebsLœffler bacillus. The patient's general condition from this time on gradually improved; the throat cleared slowly and in about two weeks had practically assumed its normal appearance.

Bacteriology. - Examinations of all the cultures taken from the throat were negative to the presence of the Klebs-Lœffler bacillus. The first culture examined was not only negative to this bacillus, but contained in pure culture a very rapidly growing, short, almost coccus-like, Gram-negative, bacillus.

The organism produced a grayish, viscid looking, slightly elevated layer on both blood serum and potato. The water of condensation showed a gray turbidity. Bouillon cultures became clouded and there was sediment formation. The organism had a slight independent motion; it did not liquefy gelatine. It produced gas in glucose-agar stab. It coagulated litmus milk and turned it pink. There was marked indol production in Dunham's peptone solution. The above characteristics served to identify the organism as a colon bacillus.

Subcutaneous inoculation of the organism with its toxin (contained in the water of condensation) caused the death of a healthy guinea pig in about fourteen hours. It is, of course, well known that the colon bacillus may cause early death by toxemia in this manner.

As was to be expected, I found the toxicity of the organism was lost after several months' cultivation in the laboratory, and thus large amounts of the organism then inoculated into a guinea pig had no effect.

Ordinarily the colon bacillus has no clinical significance and exhibits no pathogenic action. It is commonly associated, however, with other bacteria in inflammation of exposed surfaces. In this particular case, therefore, it seems probable that the organism was a secondary invader and that there must have been a primary injury to the tissue, possibly produced by the streptococcus.

The fact that the culture was pure instead of containing the great variety of organisms usually found in cultures taken from the throat was no doubt due to the great reproductive power of this organism, which enabled it to overgrow all other bacteria present. Whether or not it likewise had overcome other organisms in the throat itself is uncertain.

The picture produced in this patient by the infection was that of an acute toxemia, as shown by the high temperature, marked prostration, severe headache, chilly sensations and lumbar pains. Evidently this organism was either especially virulent or the resistance of the patient lowered, so that the tissues fell easy prey to the bacterium. How the colon bacillus came to be in the throat is a matter of conjecture. Possibly regurgitation of the stomach contents lodged the bacillus on the previously inflamed membrane. It seems more probable, however, that the infection occurred as in typhoid fever from food, water or the hands. 
The apparent improvement, after the administration of antitoxin, was probably coincidental with the successful manufacture of antibodies in sufficient numbers to protect.

The most striking feature of this case, aside from the unusual situation of the lesion, is that the offending organism, usually non-pathogenic, was in this instance strongly pathogenic.

\section{ghedical Brogregz.}

\section{PROGRESS IN THE THEORY AND PRACTICE OF MEDICINE. NEW METHODS OF DEMON- STRATING TUBERCLE BACILLI.}

\author{
BY FRANCIS W. PALFREY, M.D., BOston.
}

THE finding of tubercle bacilli is an event of such importance in the diagnosis of any case that it is natural that attention should be constantly directed toward the improvement of technical methods of attaining this result. Satisfactory as has been the Ziehl method and its modifications when it has given positive results, the frequence with which positive results have been obtained only after many examinations, and the great number of negative results in cases which are later known to be cases of tuberculosis, leave much to be desired. Thus studies of new procedures aiming to accomplish the demonstration of tubercle bacilli where the older method has failed deserve general attention.

\section{CONCENTRATION METHODS.}

For many years attempts have been made to devise means by which tubercle bacilli in sputum and other excretions may be concentrated in small compass so that when present in numbers too small to be detected readily in the simple smear they may still be detected by examination of a medium in which by special procedures they have been gathered together. These methods depend upon dissolution of the solid organic material in which the tubercle bacilli lie, followed by sedimentation. Biedert, ${ }^{1}$ in 1886 , first employed this principle by boiling the material with sodium hydrate. Muhlhäuser, ${ }^{2}$ and Czaplewski, ${ }^{3}$ in 1891, modified and improved this technic, and Camen ${ }^{4}$ in 1892 added the device since then extensively employed in laboratories of adding alcohol to reduce the specific gravity of the fluid to accelerate sedimentation. Other processes have been described by other authors. ${ }^{5}$ But these earlier methods, on account of the necessity of boiling or of much manipulation of each individual specimen, proved more laborious than was justified by their only indifferent results.

In 1908, however, two methods were published which, while similar in principle to those previously discarded, have had claimed for them great superiority in results, and more or less simplification of technic. These methods, and another observation with regard to the staining of the tubercle bacillus, to be described later, have led in the past three years to a great amount of investigation and discussion.

Chief of these is the "antiformin" method of
Uhlenhuth. "Antiformin" is the name of a proprietary fluid for use as a disinfectant, a solution of sodium carbonate and chlorinated lime, ${ }^{*}$ which Uhlenhuth found to have a remarkable power to reduce sputum and tissue to a homogeneous fluid state, with destruction of all bacteria except the tubercle bacillus. This action is obtained at room temperature, although more rapidly at higher temperatures. Cells and mucus are destroyed, leaving the bacilli free in suspension, from which they may be obtained by sedimentation, with or without centrifugalization, or by certain other devices which other authors have described.

The second method of concentration is that of Ellermann and Erlandsen, ${ }^{5}$ called by them the "double method," which, while less convenient in application than the antiformin method, has given results far in advance of older procedures, comparing favorably with those of the antiformin method.

Many articles have been published describing these newer procedures, especially the antiformin method, giving numerous modifications of technic and estimates of their value. Large numerical statistics are given in but few instances, yet enough are reported to show that the methods deserve serious consideration. Practically no unfavorable opinions have been expressed, although evidence enough has not yet been collected to justify a final estimate of their true worth.

This being the case, it is desirable to review briefly the findings of those who have most carefully studied the question. A list of authors who have written on the use of antiformin is given below. ${ }^{6-32}$ Many variations of technic are described, varying chiefly in the concentration of the antiformin solution used and in the manner of collecting the freed bacilli. The sputum or other material is treated with antiformin in varying amounts, making the concentration of the mixture all the way from $2 \%$ to nearly $50 \%$ antiformin. The mixture is shaken and allowed to stand until it is homogeneous, from thirty minutes or less to twenty-four hours, according to the strength of the solvent and to the temperature, some keeping it at room temperature, other at body temperature, others at $60^{\circ} \mathrm{C}$. and others boiling. In concentrating the freed bacilli the chief reliance is upon long centrifugalization, but a few depend upon simple sedimentation by standing, others by reduction of specific gravity by adding alcohol or acetone, while others still make use of the principle of Lang and Nitsche ${ }^{36}$ that tubercle bacilli tend to adhere to globules of the fluid hydrocarbons, benzol, ligroin, petroleum, ether or chloroform, which are emulsified in the antiformin-sputum mixture and allowed to separate, the bacilli being finally found at the zone of contact. When strong antiformin solutions are used, there is difficulty in fixing the smears. On this account some writers have washed the sediment with several changes of

* The method of preparation is given by Paterson ${ }^{23}$ as follow's: Sodium carbonate, 600; chlorinated lime, 400; Aqua dist., 4,000. Dissolve the sodium carbonate in $1,000 \mathrm{ccm}$. of the distilled water. Triturate thoroughly the chlorinated lime in the remainder of the water. Filter. Mix the two and filter again. 